EPJ Web of Conferences 71, 00042 (2014)

DOI: 10.1051/epjconf/20147100042

(C) Owned by the authors, published by EDP Sciences, 2014

\title{
Inclusive searches for SUSY from CMS
}

\author{
Arne-Rasmus Dräger for the CMS Collaboration ${ }^{1, a}$ \\ ${ }^{1}$ University of Hamburg
}

\begin{abstract}
Many searches for supersymmetry have been performed by the CMS collaboration at the LHC and in this document a selection of results of inclusive searches is presented. The searches are interpreted in scenarios of squark, gluino, chargino and neutralino production, in a variety of final states, including jets and/or leptons and missing transverse energy. The presented results are obtained from $19.5 \mathrm{fb}^{-1}$ of data taken at $\sqrt{s}=8 \mathrm{TeV}$ at the LHC in 2012.
\end{abstract}

\section{Introduction}

Up to now, all measurements from colliders are in agreement with the predictions of the standard model (SM). The recent discovery of a new scalar boson with mass of about $126 \mathrm{GeV}$ at the Large Hadron Collider (LHC) [1, 2] seems to mark another success of the SM. However, the SM can not be seen as a complete but as an effective approximation of a more complete theory. For example, the SM can not provide a candidate for dark matter which is postulated to explain astrophysical observations [3]. Also, quadratically divergent quantum loop contributions to the Higgs boson mass are making the SM a fine tuning theory. Therefore searches for physics beyond the SM are well motivated.

This proceeding reports on a selection of results of searches for physics beyond the SM in two final states. All results use the full $19.5 \mathrm{fb}^{-1}$ of data collected in pp collisions at a center of mass energy of $8 \mathrm{TeV}$ in the year of 2012 by the CMS detector [4] at the LHC.

The first search is a generic search for strongly produced new particles in multijet events with large missing transverse momentum with an explicit veto on electrons and muons [5]. The results are interpreted within the context of supersymmetric models (phenomenological MSSM) and simplified models (SMS) for direct gluino and squark pair production to obtain exclusion limits on the corresponding parameters.

The second class of searches is a combination of searches with the final state of exactly three leptons, four leptons, two same-sign leptons, two opposite-sign-same-flavor leptons plus two jets, and two opposite-sign leptons inconsistent with $Z$ boson decays [6]. In contrast to the first search the focus is mainly on electroweakly produced new particles. The results are interpreted in certain supersymmetric inspired models to to set limits on the direct production of charginos, neutralinos, and sleptons.

\footnotetext{
ae-mail: adraeger@cern.ch
} 


\section{Generic search for new physics in multijet events}

Events are selected according to these requirements: At least three jets of a $p_{\mathrm{T}}>50 \mathrm{GeV}$, a minimum transverse energy $\mathrm{H}_{\mathrm{T}}>500 \mathrm{GeV}$ which consists of the scalar sum of all jets with $p_{\mathrm{T}}>50 \mathrm{GeV}$ $|\eta|<2.5$, and a minimum of missing transverse energy $H_{\mathrm{T}}=\left|-\sum_{\text {jets }} \vec{p}_{\mathrm{T}}\right|$ of $200 \mathrm{GeV}$ are demanded. The search is carried out in 36 exclusive search regions which separate the defined minimum selection of the number of jets, $\mathrm{H}_{\mathrm{T}}$, and $\mathrm{H}_{\mathrm{T}}$. In order to reduce the amount of events arising from QCD processes where jets are heavily mismeasured events with jets $(J)\left|\Delta \phi\left(J_{n}, \mathrm{H}_{\mathrm{T}}\right)\right|>0.5 \mathrm{rad}, n=1,2$ and $\left|\Delta \phi\left(J_{3}, \mathrm{H}_{\mathrm{T}}\right)\right|>0.3 \mathrm{rad}$, are rejected. $t \bar{t}$ and $W+$ jets events can also contain large amounts of $\mathrm{H}_{\mathrm{T}}$ if leptons and neutrinos are produced therefore an explicit veto on isolated muons and electrons with $p_{\mathrm{T}}>10 \mathrm{GeV}$ is applied.

The used data sample was collected by triggering on $\mathrm{H}_{\mathrm{T}}>350 \mathrm{GeV}$ and $\mathrm{H}_{\mathrm{T}}>100 \mathrm{GeV}$.

The four dominant SM backgrounds are all measured from data: The irreducible $Z \rightarrow v \bar{v}+$ Jets background is estimated using $\gamma$-jets events exploiting their electroweak correspondence to $Z+$ Jets produced at high boson $p_{\mathrm{T}}$. The $t \bar{t}$ and $W+$ jets events pass the search selection when the $\mathrm{e} / \mu$ is not identified or isolated, or if it is out of the detector acceptance (lost-lepton background) or when a $\tau$ decays hadronically ( $\tau_{h}$ background). The lost-lepton background is estimated by reweighting events in a $\mu+$ Jets data control sample with measured lepton efficiencies. The estimation of the $\tau_{h}$ background starts from a similar $\mu+$ Jets sample where the muon is replaced by a jet sampled from a $\tau_{h}$ $p_{\mathrm{T}}$ response template obtained from the CMS simulation. The QCD multijet background is measured using a "Rebalance and Smear" $(\mathrm{R}+\mathrm{S})$ method, which predicts QCD multijet kinematics by applying measured jet response templates to smear seed events that are obtained by a procedure that produces well-balanced events from inclusive multijet data. The relative contribution of these backgrounds varies in the different search regions.

Figure 1 shows the combined predicted SM events for all backgrounds in the 36 search regions defined in $\mathrm{N}_{\mathrm{Jets}}, \mathrm{H}_{\mathrm{T}}$, and $\mathrm{H}_{\mathrm{T}}$ vs the observed events in the full $19.5 \mathrm{fb}^{-1}$ of data. The expected SM event yields are consistent with the observed number of events in data. A slight excess in data observed in the search region of $\mathrm{N}_{\text {Jets }}=[6,7], \mathrm{H}_{\mathrm{T}}=500-800 \mathrm{GeV}$, and $\mathrm{H}_{\mathrm{T}}>400 \mathrm{GeV}$ is insignificant when the probability to observe as large or larger statistical fluctuation in any of the search regions is taken into account. The further interpretation of the results is carried out for simplified models (SMS) [7, 8] of new particles.

So called simplified models which are inspired and use SUSY particle declarations are an elegant way to interpret searches for new physics and an easy way of making results transferable to various different models. Only the mass of the new produced particles and the cross-section are used to set limits. These new produced particles decay directly or via intermediate particles to quarks and a stable undetectable particle.

The limits were derived in models of $\tilde{g} \tilde{g}$ and $\tilde{q} \tilde{q}$ pairs production for a wide range of masses of the mother $(\tilde{g}, \tilde{q})$ and daughter $\left(\tilde{\chi}_{1}^{0}\right)$ particles see Fig. 2 . Other SUSY particles are assumed to be very heavy and decoupled.

PHYTHIA 6.4.24 [9] was used to generate the signal events at leading order and the cross sections are determined at the next-to-leading order in the strong coupling constant and include the resummation of soft gluon emission at the accuracy of next-to-leading-log level [10-15]. In addition the pileup pp interaction distribution was matched to the observed in data. The 95\% confidence level (CL) upper limits on the SMS signal cross section are set using the profile likelihood as a test statistic [16-18]. The results from the 36 exclusive search regions are combined into one test-statistic considering the bin-to-bin correlations of the systematic uncertainties. The observed and expected 95\% CL upper limits on the signal cross section are shown for the production of $\tilde{q} \tilde{q}$ pair with $\tilde{q} \rightarrow q+\tilde{\chi}_{1}^{0}$ in Fig. 3 , and 


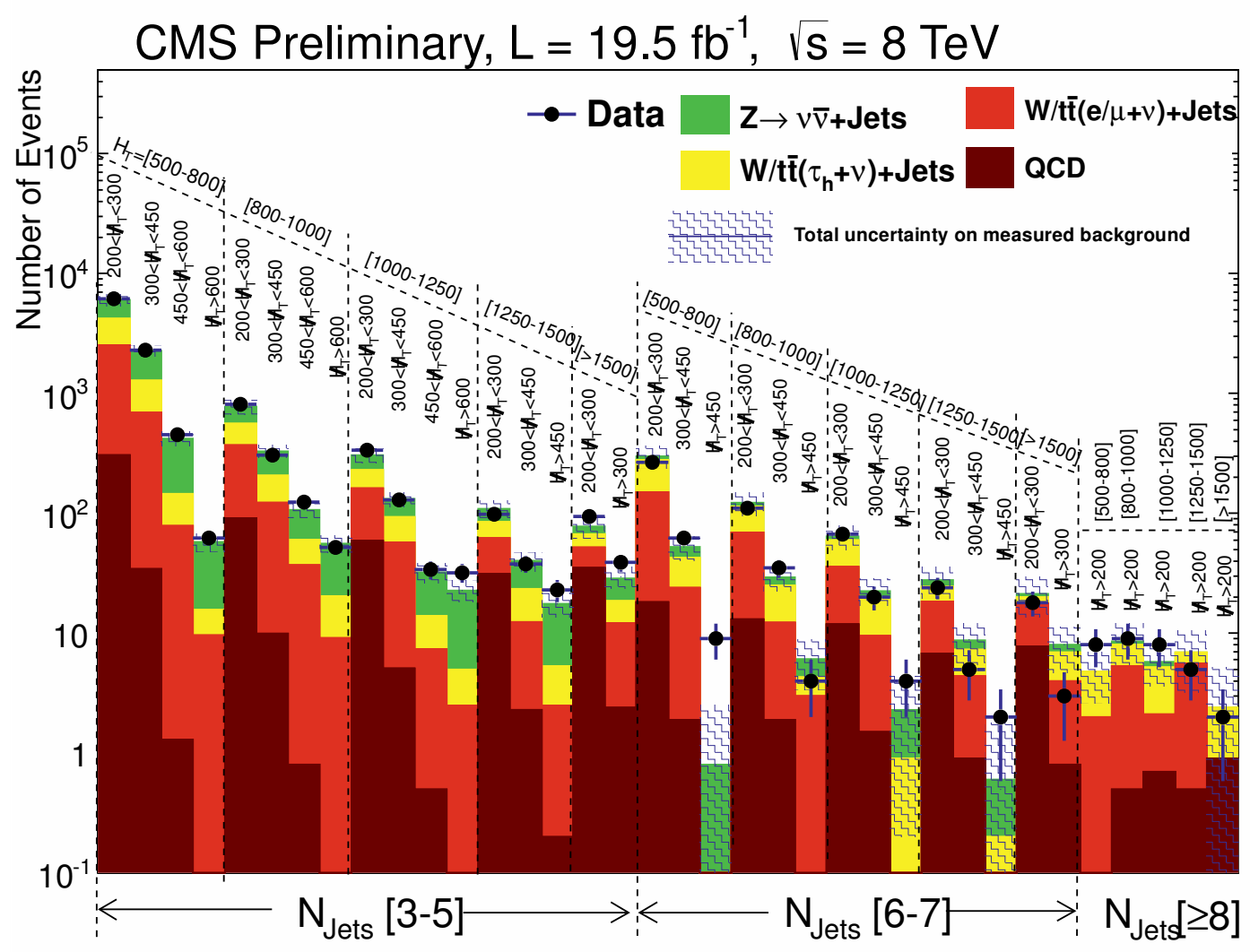

Figure 1. Summary of number of events observed in data in the 36 search regions compared to the estimated background contributions.

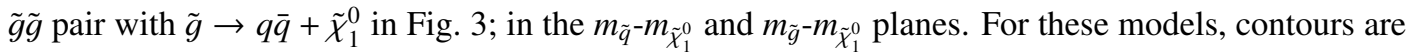
shown where the signal cross sections from NLO+NLL calculations are excluded. The exclusion contours are also presented when the signal cross section is varied by changing the renormalization and factorization scales by a factor of two and using the PDF uncertainty based on the CTEQ6.6 [19] and MSTW2008 [20] PDF sets. For $\tilde{g} \tilde{g}$ production, $m_{\tilde{g}}$ values below $1.1 \mathrm{TeV}$ for decays via light quarks are excluded. For direct $\tilde{q} \tilde{q}$ production of the first two generations of squarks $\left(\tilde{u}_{L / R}, \tilde{d}_{L / R}, \tilde{c}_{L / R}, \tilde{s}_{L / R}\right)$ values of $m_{\tilde{q}}$ below $0.75 \mathrm{TeV}$ are excluded for $m_{\tilde{\chi}_{1}^{0}}<200 \mathrm{GeV}$. If only one light squark is light enough to be accessible, $m_{\tilde{q}}$ values below $420 \mathrm{GeV}$ are excluded for $m_{\tilde{\chi}_{1}^{0}}<100 \mathrm{GeV}$.

\section{Leptonic final state}

The second search is a combination of several orthogonal final states divided by the number of leptons in the final state.

Here only the three lepton final state which consists of either an opposite-sign-same-flavor (OSSF) 

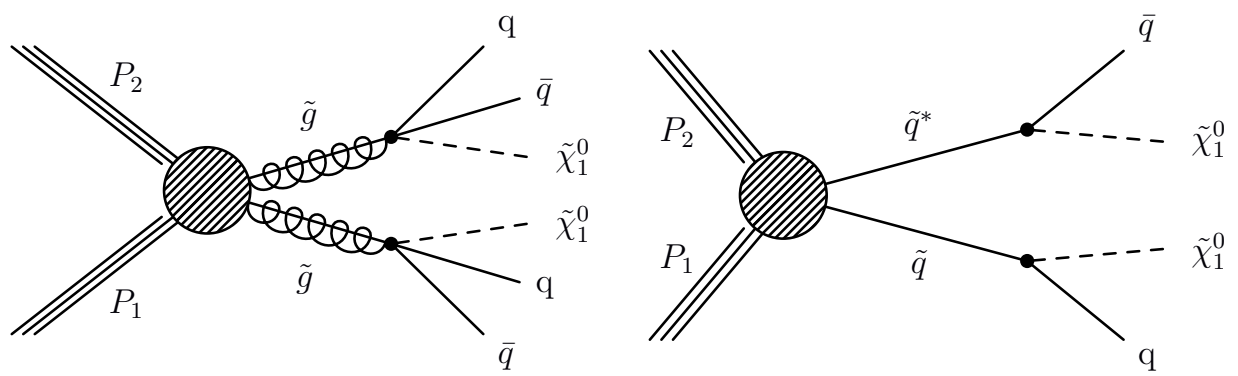

Figure 2. Feynman diagram of simplified models considered in this search: gluino pair production with $\tilde{g} \rightarrow$ $\mathrm{q} \overline{\mathrm{q}}+\mathrm{LSP}($ left) and $\tilde{q} \rightarrow q+\mathrm{LSP}$ (right).
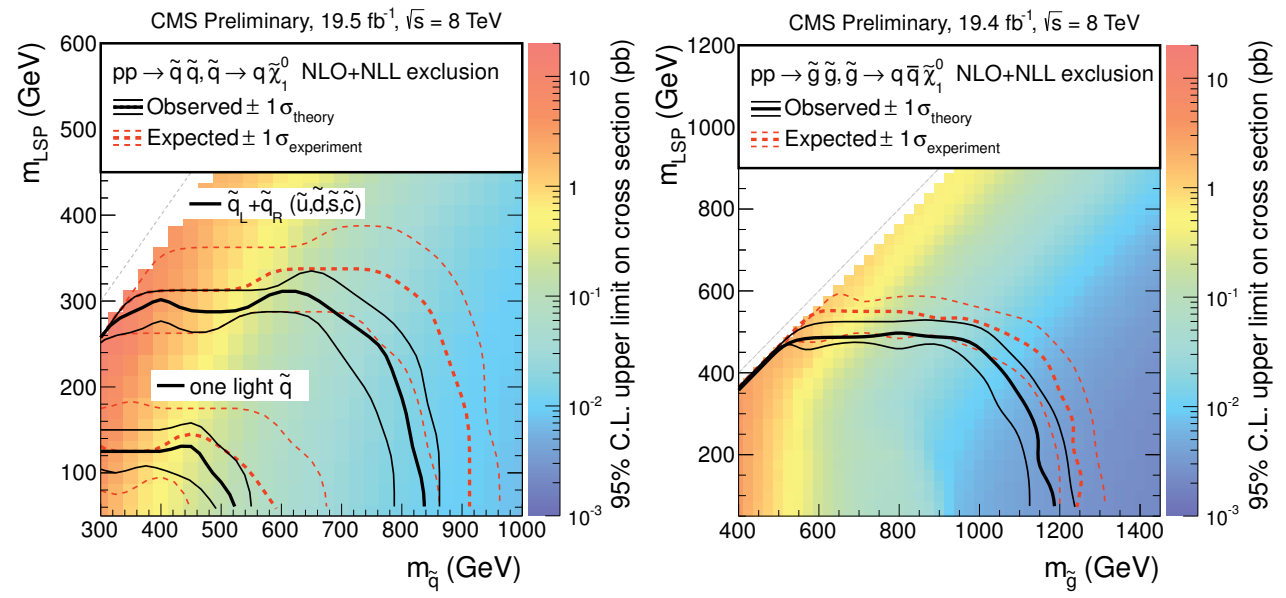

Figure 3. The observed and expected $95 \%$ C.L. upper limits on the (left) $\tilde{q} \tilde{q}$ and (right) $\tilde{g} \tilde{g}$ production crosssections in the $m_{\tilde{q}}-m_{\tilde{\chi}_{1}^{0}}$ and $m_{\tilde{g}}-m_{\tilde{\chi}_{1}^{0}}$ planes obtained with the simplified models. For the $\tilde{q} \tilde{q}$ production the upper set of curves corresponds to the scenario when the first two generations of squarks are degenerate and light, while the lower set corresponds to only one light accessible squark.

lepton pair or three leptons, will be described. The candidate events are required to have three leptons using reconstructed electrons, muons, and possibly one hadronically decaying $\tau$ leptons, of which at least one electron or muon has a $p_{\mathrm{T}}>20 \mathrm{GeV}$ and further electrons or muons have at least 10 $\mathrm{GeV}$ of $p_{\mathrm{T}}$ while the hadronic $\tau$ has to have a $p_{\mathrm{T}}>20 \mathrm{GeV}$. In addition missing transverse energy of $\#_{\mathrm{T}}>50 \mathrm{GeV}$ is demanded.

For events with an OSSF pair the invariant mass $M_{l l}$ of the lepton pair and the transverse mass $M_{T}=\sqrt{2 \cdot p_{T}^{l} \cdot H_{\mathrm{T}} \cdot(1-\cos (\Delta \Phi))}$ formed from the $Z_{\mathrm{T}}$ vector and the transverse momentum $p_{T}^{l}$ of the remaining lepton $M_{T}$ are used to characterized the events. For three-muon and three-electron events, the OSSF pair with $M_{l l}$ closer to the $\mathrm{Z}$ mass is used.

Events without an OSSF pair, which might occur in $Z \rightarrow \tau \tau$ decays, $M_{l l}$ is calculated by combining 
opposite-sign leptons and choosing the pair closest to the corresponding most probable dileptonic mass determined from $Z \rightarrow \tau \tau$ simulation.

$W Z$ production including three leptons created at the primary vertex composes the principle SM background together with $t \bar{t}$ production with two leptons from the primary vertex and one lepton which might be produced in heavy flavor decays.

The results are interpreted in exclusive search regions based on their $M_{l l},{H_{\mathrm{T}}}_{\mathrm{T}}$, and $M_{T}$ value. The OSSF dilepton pairs are divide in $M_{l l}$ bins of [75,105,Inf] GeV with further event classification for $Z_{\mathrm{T}}$ [50,100,150,200,Inf] $\mathrm{GeV}$ and $M_{T}$ regions [120,160,Inf] GeV. Fig. 4 shows $B_{\mathrm{T}}$ distributions

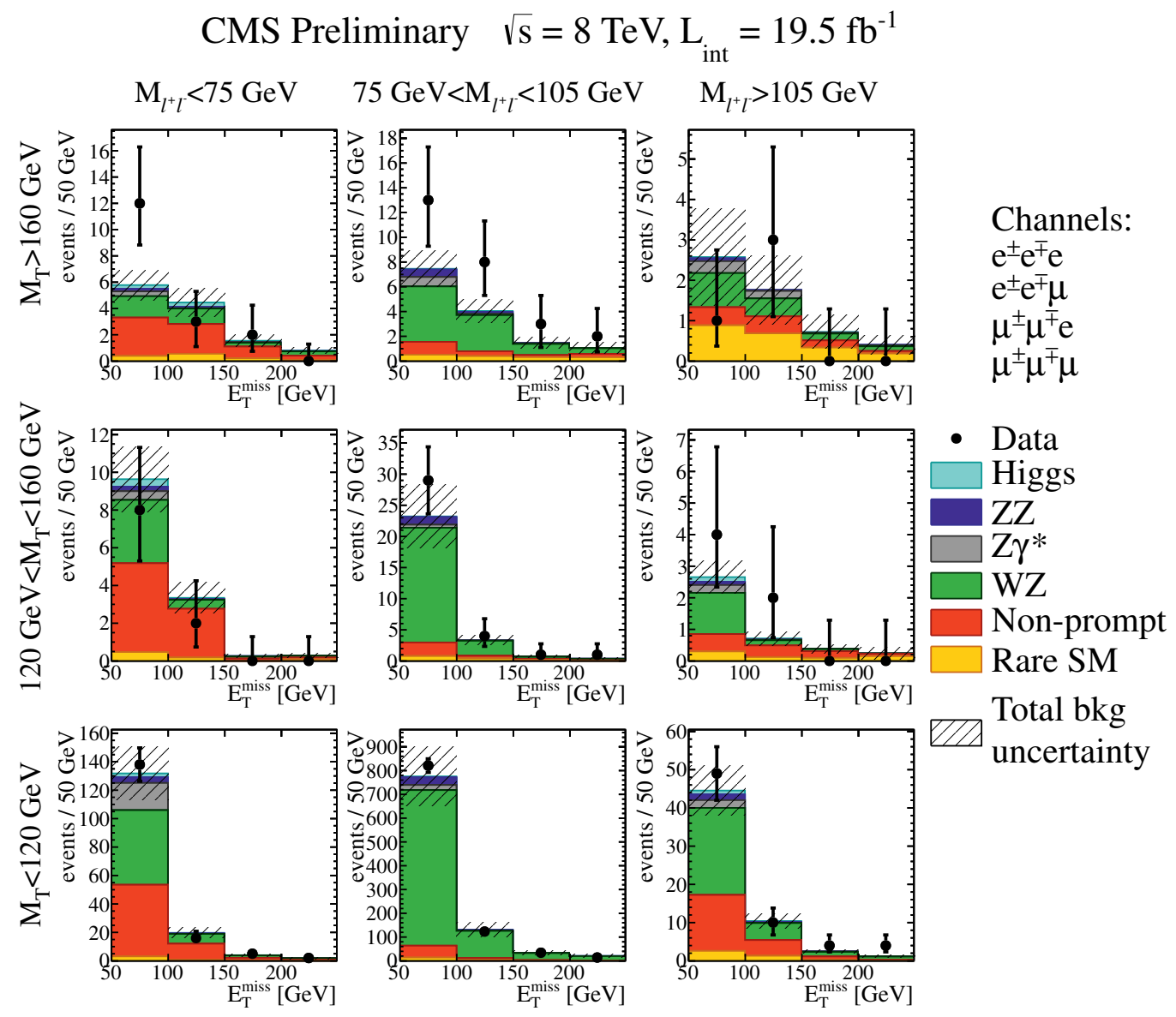

Figure 4. $Z_{\mathrm{T}}$ distribution for three-lepton events with an $e e$ or $\mu \mu$ OSSF dilepton pair, where the third lepton is either an electron or a muon. SM expectations are also shown. "Rare SM" refers to standard model processes such as $t \bar{t}+Z, t \bar{t}+W$, and triboson production. Uncertainties shown for the data are poissonian, including those for bins with zero entries.

observed in data compared to the SM expectation for the various $M_{T}$ and $M_{l l}$ regions for the ee or $\mu \mu$ OSSF pair, where the third lepton is either an electron or muon.

No statistical significant excess was observed. The results of the three lepton final state are interpreted 

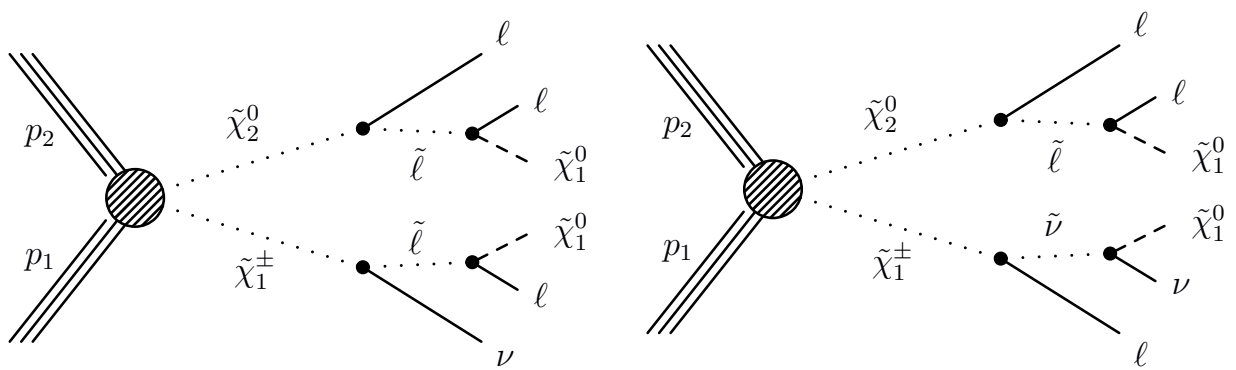

Figure 5. Chargino-neutralino pair production leading to a three-lepton final state with missing transverse energy $\#_{\mathrm{T}}$. The dotted and dashed lines indicate unstable particles and the LSP, respectively.
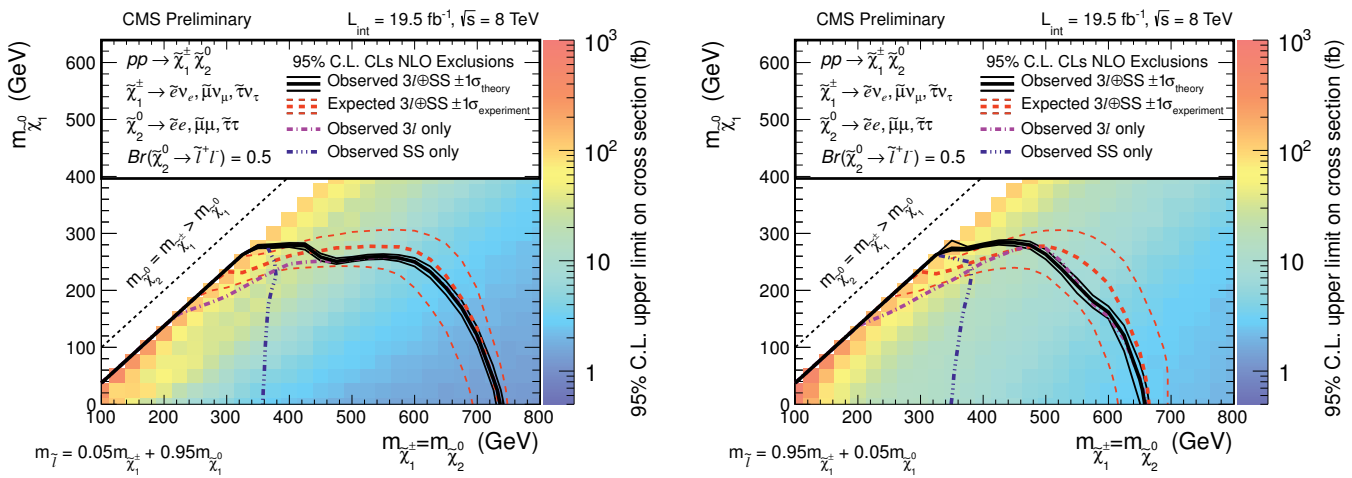

Figure 6. The shading in the $\mathrm{m}\left(\tilde{\chi}_{1}^{0}\right)$ versus $\mathrm{m}\left(\tilde{\chi}_{2}^{0}\right)$ plane indicates the $95 \% \mathrm{CL}$ upper limit on the charginoneutralino production cross section times branching fraction in the flavordemocratic scenario, for the combined analysis of the three-lepton search and the same-sign dilepton search. The contours bound the mass regions excluded at $95 \%$ CL assuming the NLO cross sections for a branching fraction of 50\%, as appropriate for the visible decay products in this scenario. The contours based on the observations are shown for the combination; in addition, the expected combined bound is shown. Other contours show separate mass exclusions for the threelepton search and the same-sign dilepton search alone.

combined with the same-sign dilepton search in terms of simplified models. Fig. 5 illustrate the two SMS topologies used for interpreting the three lepton and two lepton same-sign search results. The obtained exclusion limits are shown in Fig. 6. The 95\% CL upper limit on the cross section times branching fraction in the $\mathrm{m}\left(\tilde{\chi}_{1}^{0}\right)$ versus $\mathrm{m}\left(\tilde{\chi}_{2}^{0}\right)$ plane in the flavor-democratic scenario (the decay probability to all lepton flavors is the same) is depicted in this figure for two values of $x_{\tilde{l}}=0.05$ and 0.95 with $x_{\tilde{l}}$ determining the slepton mass in terms of the involved chargino and neutralino masses see Eq. 1 .

$$
m_{l}=m_{\tilde{\chi}_{1}^{0}}+x_{\tilde{l}} \cdot\left(m_{\tilde{\chi}_{1}^{ \pm}}-m_{\tilde{\chi}_{1}^{0}}\right)
$$




\section{Conclusion}

Many inclusive searches have been performed by CMS looking for heavy and weakly produced new supersymmetric particles in various final states of multijets, high missing transverse energy in the full hadronic and also leptonic final state. $19.5 \mathrm{fb}^{-1}$ of $8 \mathrm{TeV}$ pp data collected in 2012 have been analyzed. No excess over the standard model expectation has been observed. The absence of an excess has been interpreted in terms of simplified models and phenomenological MSSM. The presented CMS searches are able to exclude gluino masses up to 1.1-1.2 TeV and squark masses below $0.78 \mathrm{TeV}$ within the studied models for $\tilde{\chi}_{1}^{0}$ masses below $200 \mathrm{GeV}$ and chargino masses up to $500 \mathrm{GeV}$ with neutralino masses below $200 \mathrm{GeV}$.

\section{References}

[1] CMS Collaboration, "Observation of a new boson at a mass of $125 \mathrm{GeV}$ with the CMS experiment at the LHC”, Phys. Lett. B 716 (2012) 30, doi:10.1016/j.physletb.2012.08.021, arXiv:1207.7235.

[2] ATLAS Collaboration, "Observation of a new particle in the search for the Standard Model Higgs boson with the ATLAS detector at the LHC", Phys. Lett. B 716 (2012) 1, doi:10.1016/j.physletb.2012.08.020, arXiv:1207.7214.

[3] J. L. Feng, "Dark Matter Candidates from Particle Physics and Methods of Detection", Ann. Rev. Astron. Astrophys. 48 (2010) 495, doi:10.1146/annurev-astro-082708-101659, arXiv:1003.0904.

[4] S. Chatrchyan et al. [CMS Collaboration], "The CMS experiment at the CERN LHC", JINST 3:S08004,2008.

[5] CMS Collaboration, "Search for New Physics in the Multijets and Missing Momentum Final State in Proton-Protn Collisions at $\sqrt{s}=8 \mathrm{TeV}$ ", CERN Document Server CMS-PAS-SUS-13012, https://cds.cern.ch/record/1563156

[6] CMS Collaboration, "Search for electroweak producti of charginos, neutralions, and sleptons using leptonic final states in pp collisions at $\sqrt{s}=8 \mathrm{TeV}$ ", CERN Document Server CMS-PASSUS-13-017, http://cds.cern.ch/record/1596278

[7] Alwall, Johan and Schuster, Philip and Toro, Natalia, "Simplified Models for a First Characterization of New Physics at the LHC", Phys. Rev. D 79 (2009) 075020, doi:10.1103/PhysRevD.79.075020, arXiv:0810.3921.

[8] CMS Collaboration, "Interpretation of searches for supersymmetry with simplified models", CMS-SUS-11-016 (2013), arXiv:1301.2175.

[9] Sjöstrand, Torbjorn and Mrenna, Stephen and Skands, Peter Z., "PYTHIA 6.4 Physics and Manual”, JHEP 05 (2006) 026, doi:10.1088/1126-6708/2006/05/026, arXiv:hep-ph/0603175.

[10] Beenakker, Wim and Höpker, R. and Spira, M. and Zerwas, P. M., "Squark and gluino production at hadron colliders", Nucl. Phys. B 492 (1997) 51, doi:10.1016/S0550-3213(97)00084-9

[11] Kulesza, Anna and Motyka, L., "Threshold resummation for squark-antisquark and gluino-pair production at the LHC", Phys. Rev. Lett. 102 (2009) 111802, doi:10.1103/PhysRevLett.102.111802

[12] Kulesza, Anna and Motyka, L., "Soft gluon resummation for the production of gluino-gluino and squark-antisquark pairs at the LHC", Phys. Rev. D 80 (2009) 095004, doi:10.1103/PhysRevD.80.095004

[13] Beenakker, Wim and Brensing, Silja and Krämer, Michael and Kulesza, Anna and Laenen, Eric and Niessen, Irene, "Soft-gluon resummation for squark and gluino hadroproduction", J. High Energy Phys. 12 (2009) 041, doi:10.1088/1126-6708/2009/12/041 
[14] Beenakker, Wim and Brensing, Silja and Krämer, Michael and Kulesza, Anna and Laenen, Eric and Motyka, Leszek and Niessen, Irene, "Squark and gluino hadroproduction", Int. J. Mod. Phys. A 26 (2011) 2637, doi:10.1142/S0217751X11053560

[15] M. Kramer et. al., "Supersymmetry production cross sections in ppcollisions at $\sqrt{s}=7 \mathrm{TeV}$, arXiv: 1206.2892

[16] Alex L. Read, "Presentation of search results: the CLs technique", Journal of Physics G: Nucl. Part. Phys. 28 (2002) 2693, doi:10.1088/0954-3899/28/10/313

[17] Tom Junk, "Confidence level computation for combining searches with small statistics", Nuclear Instruments and Methods A434 (1999) 435, doi:10.1016/S0168-9002(99)00498-2, arXiv:hepex/9902006

[18] ATLAS and CMS Collaborations, "Procedure for the LHC Higgs boson search combination in Summer 2011", ATL-PHYS-PUB-2011-011, CMS NOTE-2011/005, https://cdsweb.cern.ch/record/1379837

[19] Nadolsky, Pavel M. and others, "Implications of CTEQ global analysis for collider observables", Phys. Rev. D 78 (2008) 013004, doi:10.1103/PhysRevD.78.013004, arXiv:0802.0007

[20] Martin, A.D. and others, "Parton distributions for the LHC", Eur. Phys. J. C 63 (2009) 189, doi:10.1140/epjc/s10052-009-1072-5, arXiv:0901.0002 